Rivera Otero, J.M., Lagares Diez, N., Jaráiz Gulías, E., López-López, P.C. (2021): Emociones y engagement en los mensajes digitales de los candidatos a las elecciones generales de 2019. Cultura, Lenguaje y Representación, Vol. XXVI, 229-245

ISSN $1697-7750 \cdot$ E-ISSN 2340-4981

DOI: http://dx.doi.org/10.6035/clr.5844

\title{
Emociones y engagement en los mensajes digitales de los candidatos a las elecciones generales de 2019
}

Emotions and engagement in the digital messages of the candidates for the 2019 general elections

JosÉ MANUEL RiVERA OTERO

UNIVERSIDAD DE SANTIAGO DE COMPOSTELA

HTTPS://ORCID.ORG/0000-0003-0306-1768

NiEVES LAGARES DiEZ

UNIVERSIDAD DE SANTIAGO DE COMPOSTELA

HTTPS://ORCID.ORG/0000-0001-5304-0581

ERIKA JARÁIZ GULÍAS

UNIVERSIDAD DE SANTIAGO DE COMPOSTELA

HTTPS://ORCID.ORG/0000-0003-2382-6713

PAULO CARLOS LÓPEZ-LÓPEZ

UNIVERSIDAD DE SANTIAGO DE COMPOSTELA

HTTPS://ORCID.ORG/0000-0002-8101-7976

RESUMEN: Las redes sociales se han convertido en los últimos años en una importante plataforma de comunicación a través de la que los candidatos promueven el compromiso político de sus electores. Una de las variables que podría influir en esa capacidad de generar engagement a través de las redes sociales es la utilización de mensajes con una importante carga emocional, tanto positiva como negativa. Nuestra investigación tiene como objetivo profundizar en el análisis de dicha relación entre el compromiso político y las emociones en las redes sociales, mediante el Análisis de Sentimientos en los tuits de los principales candidatos en las elecciones generales de abril y noviembre de 2019 en España. Para ello recurrimos a la técnica de extracción de textos y su análisis con el software Linguistic Inquiry and Word Count (LIWC), poniendo en 
relación el nivel de presencia de emociones positivas y negativas, con el nivel de compromiso alcanzado por los candidatos durante la campaña electoral. Nuestro análisis contribuye a demostrar la existencia de relación entre la carga emocional del discurso en las redes sociales y el nivel de engagement que genera, aunque este parece estar mediatizado además por la agenda política y la estrategia de campaña de los partidos. Comprobamos además que la relación entre discurso emocional y engagement hacia un líder no es exclusivamente endógena, sino que puede obedecer también a elementos exógenos debido a la presencia de comunidades digitales compartidas.

Palabras clave: compromiso político, redes sociales, Twitter, emociones, análisis de sentimientos.

ABSTRACT: In recent years, social networks have become an important communication platform through which candidates promote the political engagement of their constituents. One of the variables that could influence this ability to generate engagement through social networks is the use of messages with a significant emotional charge, both positive and negative. Our research aims to delve into the analysis of this relationship between political engagement and emotions in social networks, through the Sentiment Analysis in the tweets of the main candidates in the general elections of April and November 2019 in Spain. For this we resort to the technique of text extraction and its analysis with the Linguistic Inquiry and Word Count (LIWC) software, relating the level of presence of positive and negative emotions, with the level of engagement reached by the candidates during the electoral campaign. Our analysis helps to demonstrate the existence of a relationship between the emotional charge of the discourse on social networks and the level of engagement it generates, although this seems to be also mediated by the political agenda and the campaign strategy of the parties. We also verify that the relationship between emotional discourse and engagement towards a leader is not exclusively endogenous, but may also be due to exogenous elements due to the presence of shared digital communities.

Key words: political engagement, social network, Twitter, emotions, Sentiment Analysis.

\section{INTRODUCCIÓN}

El aumento de las investigaciones sobre el lenguaje emocional en los discursos políticos se ha trasladado, en los últimos años, a las redes sociales, que se han convertido en la herramienta fundamental de estrategia de las campañas online y complemento a las actividades offline de los partidos, llegando incluso a facilitar la participación de determinados colectivos que en otras condiciones no participarían políticamente, especialmente los jóvenes (Aldrich et al., 2016).

Esta participación en las redes sociales es una expresión del nivel de compromiso político que algunos de sus seguidores adquieren con los partidos y líderes, pero todavía no está claro cuáles son los condicionantes de la generación de este compromiso. La presencia de emociones en los mensajes de los políticos y sus seguidores ha sido 
relacionada con un aumento del engagement en las redes sociales (Gerbaudo el al., 2019), pero los estudios al respecto son todavía escasos. El objetivo de este artículo es profundizar en el análisis del componente emocional de los mensajes de los políticos en las redes sociales, con el fin de analizar si generan más engagement las emociones positivas o negativas, y si se trata esta de una relación exclusivamente entre un líder y sus seguidores, o existe interacción entre los seguidores de diferentes líderes y los mensajes emitidos por estos.

Para llevar a cabo nuestra investigación hemos escogido como estudio de caso las últimas dos elecciones generales celebradas en nuestro país, y la discusión política acontecida en Twitter durante ambas campañas electorales. Las elecciones generales de 2019 en España (abril y noviembre), constituyen una situación excepcional en nuestra democracia por el alto grado de polarización del sistema de partidos y el hecho de que en un mismo año se repitan unas elecciones debido a la incapacidad de alcanzar un acuerdo de gobierno entre los diferentes partidos políticos.

Esta investigación profundiza en la literatura científica sobre las emociones y el compromiso político en las redes sociales, para a partir de ella establecer e interpretar las relaciones entre el nivel de engagement de los políticos en cada día que de campaña y la proporción de sentimientos positivos y negativos presentes en sus tuits.

\section{EL ENGAGEMENT POLÍTICO EN LAS REDES SOCIALES}

La evidencia sugiere que el uso de las redes sociales favorece la participación política y fomenta el compromiso de los ciudadanos con actividades cívicas (Gil de Zúñiga et al., 2012).

El concepto de compromiso político (engagement) es, sin embargo, a menudo un concepto controvertido y de difícil definición (Ekman y Amnå, 2012), dando lugar a lecturas que van desde ser entendido como sinónimo de participación por una parte de la literatura especializada (Aldrich et al., 2016), abarcando comportamientos, procesos cognitivos y actitudes relacionados con la política, tales como estar interesado por la política, en la búsqueda de información política o estar identificado con un partido político (compromiso cognitivo); hasta contactar con políticos, asistir y participar en reuniones políticas y participar electoralmente (compromiso activo) (Eckstein, Noack y Gniewosz, 2012). Algunos autores lo consideran un tipo específico de compromiso cívico, siendo éste un medio para participar e influir en la vida de una comunidad, mientras el compromiso político buscaría, específicamente, influir en los problemas, los sistemas y las estructuras políticas (McCartney et al. 2013). A esta dificultad en su definición, es necesario añadir la complejidad de la medición del compromiso político en las redes sociales, dando lugar al debate entre aquellos que defienden su naturaleza multidimensional, diferenciando, por ejemplo, entre un compromiso activo y un compromiso pasivo (Gibson y Cantijoch, 2013; Cantijoch et al., 2015), frente a los que defienden que, a pesar de tener múltiples manifestaciones, se trata de un fenómeno unidimensional (Krueger, 2002).

El estudio del engagement, surge a partir de los estudios sobre el compromiso y la participación política offline (Rosenstone y Hansen, 1993; Verba et al., 1995), y fue la extensión del uso de las redes sociales para informarse y participar políticamente la que propició, muy pronto, el interés por el estudio del compromiso político online (Anduiza, et al., 2012), hasta el punto de que los medios digitales han sido vistos por algunos autores como la solución a los crecientes problemas de falta de compromiso con la política (Macková, 2015), en forma de desafección, escepticismo o desconfianza (Mahgallaes, 2005) en las democracias consolidadas. El atractivo de las redes para el estudio del 
engagement proviene de que los medios digitales permiten el contacto personalizado y directo de partidos y líderes políticos con sus votantes, mediante la construcción de comunidades digitales y redes sociales (Lilleker, 2013), que favorecen el desarrollo y mantenimiento de la confianza interpersonal (Ellison et al., 2007), y fomentan la exposición de los ciudadanos a información política de carácter movilizador (Tang y Lee, 2013). Lo cierto es que los medios digitales, han generado oportunidades para el compromiso político que no eran posibles anteriormente (Norris, 2001), ya facilitando el encuentro de los ciudadanos con la información, muchas veces de forma accidental (Gil de Zúñiga et al., 2012; Tang y Lee, 2013), sin buscarla activamente, ya haciendo más accesible la movilización de los votantes por parte de los políticos, los militantes y otros ciudadanos $^{1}$, sobre todo de los jóvenes (Aldrich et al., 2016), muchos de los cuales no habrían sido invitados a participar a través de los medios de movilización tradicionales (Verba et al., 1995).

Frente a la visión pesimista sobre el impacto negativo que la aparición de internet y los medios digitales podrían tener en la participación democrática (Mozorov, 2011), la mayoría de las investigaciones enfatizan la existencia de una relación positiva entre el uso de redes sociales y la participación política online (Skoric, 2016) e incluso con la participación offline (Gibson, 2013), apuntando a la existencia de cierta interdependencia entre ambas. Sin embargo, que el acceso a internet sea interpretado en su capacidad para atraer a nuevos participantes, previamente inactivos, a la vida política y cívica (Stanley y Weare, 2004; Vaccari, 2016) provoca, también, visiones contrapuestas en los que defienden que la capacidad movilizadora de las nuevas tecnologías es limitada, ya que únicamente contribuirían a reforzar el engagement de aquellos que ya estaban involucrados previamente (Bimber, 1999, Norris, 2003).

Las lecturas de esta relación son muy variadas, desde los que afirman que el uso de los medios digitales no está relacionado con la participación política offline (Baumgartner y Morris, 2010), hasta los que defienden una relación positiva (Bode, 2012); o los que afirman (Bimber, et al., 2002) que son los individuos políticamente comprometidos los que utilizan los medios digitales en mayor medida que aquellos que no lo están. En el fondo, porque todos los análisis están basados en casos en los que el lenguaje no puede ser analizado de forma neutra sino por relación a la estrategia política que conduce.

En definitiva, no hay acuerdo en la literatura especializada sobre la capacidad de las redes sociales para promover el compromiso político, pero tampoco hay evidencias sólidas de lo contrario (Skoric, 2015) más allá de meros casos singulares. Los metaanálisis de Boulianne (2015) muestran que tanto el uso de las redes sociales, como de internet en general (Boulianne, 2009), se asocian positivamente con mayores niveles de participación política, mientras Skoric (2015), por su parte, limita el alcance de su hallazgo a la relación positiva existente entre el uso de los medios digitales para informarse y la participación política.

Las primeras investigaciones se centraban en la utilización de internet (Bimber, 2001; Krueger, 2002), los e-mails (Krueger, 2010) e incluso los mensajes de texto (sms) (Kenski et al., 2010; Malhotra et al., 2011) en las campañas electorales, especialmente en la victoria de Obama en 2008, como forma de contacto y de movilización política, generando, en última instancia el compromiso político de los ciudadanos. Sin embargo, la generalización del uso de los medios digitales, en su acepción más amplia, dio lugar a la aparición de numerosas investigaciones centradas en su papel como herramientas de

${ }^{1}$ En concordancia con el modelo two-step flow (Norris y Curtice, 2008) los partidos y sus gestores utilizarían internet para influenciar a activistas online, de modo que estos a su vez alcancen a un público más general. 
comunicación entre partidos políticos y líderes con los votantes (Bond et al., 2012; Gerodimos y Justinussen, 2015), con Facebook y Twitter como las más utilizadas, y por lo tanto las más estudiadas. Más recientemente, la atención se ha centrado en los servicios de mensajería instantánea, como WhatsApp, cuyas características permiten nuevas funcionalidades, como compartir mensajes multimedia, y favorecen el diálogo y la discusión en la red (Valenzuela et al. 2019) proporcionando, a diferencia de Twitter y Facebook, un entorno controlado y relativamente privado para las conversaciones políticas (Valeriani y Vaccari 2017).

\section{EMOCIONES Y ENGAGEMENT POLÍTICO EN LAS REDES SOCIALES}

La presencia de los candidatos en las redes sociales posibilita las conversaciones online en torno a los issues políticos. De este modo, un uso expresivo de los medios digitales promueve la comunicación interpersonal y el establecimiento de una dinámica dialógica favoreciendo, a su vez, el compromiso político (Jung, Kim y Gil de Zúñiga, 2011). Para Wise, Bolls y Shaefer (2008), el uso activo de los medios digitales, como la búsqueda, la compartición o el comentario de información online, generan cognitivamente más compromiso que la utilización de medios tradicionales de comunicación, y aun estando de acuerdo en la importancia del diálogo como elemento favorecedor del engagement político, es en esa atribución de un carácter exclusivamente cognitivo al mismo, dónde nuestro planteamiento difiere del enfoque de buena parte de la literatura especializada existente.

Los estudios sobre el compromiso político a través de las redes sociales, se han centrado prioritariamente en el análisis de elementos de carácter cognitivo, sin embargo, algunos autores extienden el concepto para, sin dejar al margen los comportamientos, defender la existencia de una doble dimensión psicológica en la que intervienen cogniciones y emociones (Barrett, 2014), haciendo del compromiso político una condición previa para la participación, es decir, un prerrequisito de ésta (Dahlgren, 2009). Esta lectura, que asumimos, permite hablar de compromiso cognitivo (conocimiento político, atención a los medios, conversaciones sobre temas políticos) o de compromiso emocional (intensidad de sentimientos hacia asuntos políticos o cívicos), pudiendo producirse este último, sin la necesidad de tener comportamientos comprometidos. En cualquier caso, tanto la conexión como la desconexión deben tener un fundamento motivacional (Dahlgren, 2009) y es ahí donde enraíza el engagement emocional. Por eso, cuando la teoría democrática defiende el compromiso político de los ciudadanos desde presupuestos exclusivos de la racionalidad y olvida la presencia de lo afectivo, emocional, pasional, acaba ignorando, como ha señalado Hall (2005) que la motivación sin afecto es imposible de entender y de explicar.

El predominio de la razón ha condicionado la mayor parte de las investigaciones sobre comportamiento y comunicación política, sin embargo, en las últimas dos décadas, ha ido creciendo el interés por el papel que juegan las emociones en nuestras decisiones políticas, favorecido por el desarrollo de nuevos métodos y técnicas de investigación, incluidos algunos softwares, que permiten el abordaje de una materia de gran complejidad. De este modo, el reconocimiento de la importancia de los afectos y las emociones en el procesamiento de la información (Zillman, 1971; Redlawsk, 2006; Neuman et al., 2018) ha tenido como consecuencia la proliferación de investigaciones de análisis de sentimientos en los discursos, especialmente en el ámbito de las redes sociales. Para Brady y otros (2017), la utilización del lenguaje emocional en las redes sociales aumenta su capacidad de difusión entre las distintas comunidades digitales, favoreciendo el Social Sharing Emotional (Bazarova et. al, 2015). Esto nos permite interpretar las 
emociones, desde un enfoque constructivista, como socialmente compartidas (Gordon, 1981), un proceso de convergencia emocional o un estado de ánimo construido que se intensifica a medida que se acerca el final de la campaña electoral (Jaráiz-Gulías, PereiraLopez y Rivera Otero, 2021).

El estudio de la relación entre el uso de las redes sociales y el engagement político se ha desarrollado, a menudo, en torno a lo acontecido durante las campañas electorales en múltiples países (Bond et al., 2012; Gerbaudo, 2019), especialmente en Estados Unidos. Los políticos y sus equipos implementan estrategias de campaña específicas para las redes sociales (Kreis, 2017; Lee y Xu, 2018), entre las que destaca la definición de un emotional framing, (Nabi, 2003) en el que los mensajes específicos cobran sentido y oportunidad. Siguiendo a Entman (1993), en su análisis de la construcción de marcos interpretativos en las campañas de Trump y Clinton de 2016, Sahly y otros (2019) examinaron el nivel de compromiso político de la audiencia en los medios digitales generado en base a los efectos de los marcos emocionales utilizados por los candidatos, concluyendo que junto a los marcos interpretativos de conflicto y moralidad, los marcos emocionales (positivos y negativos), contribuyen a incrementar el engagement de la audiencia de Twitter y Facebook.

Aunque la literatura que relaciona el uso de las emociones en las redes sociales y la generación de compromiso político online es relativamente escasa, se ha generado un amplio consenso en la afirmación de que las emociones, particularmente intensas, tanto positivas como negativas, en las comunicaciones online, son capaces de generar un mayor nivel de compromiso político. Las dificultades metodológicas en el abordaje de esta cuestión han hecho que muchas de estas investigaciones se centren en algunas acciones y actitudes específicas relacionadas con el engagement político, como la viralización de los mensajes en las redes (Berger y Milkman, 2012), o su capacidad de generar simpatía (Cvijikj y Michahelles, 2013), o incluso en la capacidad de algunos mensajes de generar más likes que otros (Nave et al., 2018). Existe la idea, ampliamente compartida, de que la comunicación política en las redes sociales está dominada por contenidos negativos, sobre todo durante las campañas electorales (Savoy, 2017), atribuyéndole un mayor éxito electoral a las estrategias de comunicación con una fuerte carga emocional negativa (Ceron y d'Adda, 2015; Liu y Lei, 2018). En sentido opuesto, Stieglitz and Dang-Xuan (2013) encontraron una correlación positiva entre la carga emocional de los mensajes emitidos y el número de retuits obtenidos, y Berger y Milkman (2012) señalaron que el contenido positivo en las redes sociales es más susceptible de hacerse viral, aunque también señalan que las publicaciones con contenido emocional, ya sea positivo o negativo, que desencadenan una respuesta emocional intensa, se hacen más virales. En su análisis de las emociones en Facebook en las elecciones de 2017 en el Reino Unido, Gerbaudo y otros (2019) encuentran una fuerte correlación entre los contenidos positivos en las redes sociales de los candidatos (positive posting) y un alto nivel de engagement de los usuarios, de modo que las estrategias de campaña enfocadas en provocar reacciones emocionales positivas son capaces de generar más motivación y mayores niveles de compromiso político entre los usuarios de las redes sociales, que las estrategias negativas.

En general, las investigaciones sobre engagement y emociones se refieren a la capacidad de los líderes de generar compromiso político con su audiencia mediante la emisión de mensajes con contenido emocional, casi siempre en relación a las emociones que expresan hacia el propio líder. Nuestro propio trabajo previo sobre el impacto de las emociones en el liderazgo y en el voto nos ha mostrado que dicho impacto no se construye solo sobre la expresión de emociones positivas hacia el líder o el partido que se vota, sino también en la expresión de emociones negativas hacia los otros partidos o líderes. Y por eso, en una dinámica polarizada como la que se da en estas elecciones, nuestro análisis se 
orienta a buscar si es posible establecer una conexión entre el engagement hacia un candidato y la expresión de emociones negativas hacia el resto de los candidatos; y de producirse, analizar si estas conexiones tienen lugar dentro del propio espacio ideológico o fuera del mismo. Conforme a esta lectura establecemos las siguientes hipótesis que nos servirán de punto de partida para nuestra investigación.

H1: El engagement político responde a criterios de la limitada conectividad entre los usuarios alineados a la izquierda o a la derecha del espectro ideológico.

H2: El vínculo entre un líder y sus seguidores no se expresa sólo a través de las emociones positivas hacia él, sino también mediante la expresión de emociones negativas hacia otros líderes, y esa negatividad también genera engagement.

H3: Los partidos de extrema derecha tienen mayor capacidad de generar engagement, especialmente a través de emociones negativas.

\section{EMOCIONES Y ENGAGEMENT EN EL TWITTER DE LOS CANDIDATOS EN LAS ELECCIONES GENERALES DE 2019}

Las elecciones generales de 2019 en España constituyen un objeto de estudio especialmente interesante para contrastar nuestras hipótesis debido, entre otras cuestiones, a la alta polarización del sistema de partidos en el momento en que se celebran y al ascenso de la extrema derecha. El contexto político es especialmente complejo debido a la llegada al poder de Pedro Sánchez tras la moción de censura a Mariano Rajoy en 2018, y la convocatoria de elecciones menos de un año después, debido a la incapacidad de aprobar un presupuesto de consenso. Las elecciones de abril son las terceras elecciones generales en España en menos de cuatro años, y las elecciones de noviembre son las segundas que se convocan tras la imposibilidad de formar gobierno. Ambas convocatorias electorales, especialmente la de noviembre, suponen además el gran éxito de VOX, que pasa de no obtener ningún escaño en las elecciones generales de 2016 , a tener un $10 \%$ y un $15 \%$ de los votos en abril y en noviembre, respectivamente. Este dato resulta relevante para nuestra investigación, ya que, según la literatura especializada, los partidos de extrema derecha parecen haber sido especialmente exitosos en el uso de las redes sociales en sus campañas electorales (Enli, 2017), sobre todo en la utilización de contenido negativo (Groshek y Koc-Michalska, 2017).

En la mayoría de las investigaciones sobre el compromiso político generado en las redes, la forma de medir dicho compromiso es, o bien a través de la realización de encuestas ad-hoc, o utilizando como indicador el nivel de audience engagment ${ }^{2}$, es decir, el nivel de participación de los usuarios e interactividad en tiempo real en las redes sociales. En nuestro análisis adoptamos este último enfoque, analizando el contenido emocional de los mensajes emitidos en Twitter por los candidatos a las elecciones generales de abril y noviembre de 2019, y poniéndolo en relación con el nivel de engagement obtenido. Twitter es una red social que sirve como plataforma para la discusión y participación política (Vaccari et al., 2013) y el desarrollo de estrategias en las campañas electorales, siendo considerada una red social más polarizada que Facebook (Yarchi, Baden y Kligler-Vilenchik, 2020), por lo que su elección para nuestro caso de análisis resulta especialmente adecuada. En Twitter los usuarios suelen estar condicionados por una exposición selectiva, leyendo casi en exclusiva las publicaciones de iguales con ideas afines (Feller et al., 2011), de modo que los mensajes emitidos en

\footnotetext{
2 Twitter define el engagement como el número de veces que un usario interactua con un tuit, incluyendo clicks, retweets, replies, follows, likes, links, cards, hashtags, contenido multimedia, nombre de usuario o foto de perfil.
} 
esta red social alcanzan generalmente audiencias previamente comprometidas. En este sentido, los datos de Twitter han sido utilizados para estudiar la polarización política.

El abordaje metodológico del análisis de las emociones en redes sociales se ha llevado a cabo fundamentalmente mediante la utilización de dos técnicas diferentes: el aprendizaje automático supervisado y los métodos no supervisados, que utilizan léxicos con palabras puntuadas en función de sus distintos valores de polaridad u orientación semántica (positivo, negativo y neutro). Esta última técnica denominada análisis de sentimientos (D’Andrea, 2015), se sustenta en las teorías emocionales de valencia única (Fishbein y Ajzen 1975), y ha sido utilizada con éxito en numerosas investigaciones en los últimos años (Liu y Lei, 2018; Bhattacharya et al., 2015; Murthy, 2015; Jaráiz et al., 2021), para analizar tanto los discursos emitidos por los políticos, como las reacciones que estos provocan en el electorado en las redes sociales. Esta es la técnica seleccionada para nuestro análisis de los tuits emitidos por los principales candidatos y sus seguidores en las campañas electorales de las elecciones generales de abril y noviembre de 2019 en España. A tal efecto, mediante la técnica de extracción de textos, hemos realizado el análisis de sentimiento, es decir, el análisis de la presencia de emociones positivas y negativas, en los tuits emitidos desde las cuentas de los principales candidatos ${ }^{3}$ en el periodo de las dos campañas electorales más recientes en España (del 12 al 25 de abril y del 1 al 8 de noviembre de 2019) $)^{4}$. A tal efecto, hemos empleado el software Linguistic Inquiry and Word Count (LIWC), cuya efectividad ha sido previamente testada en múltiples investigaciones propias y ajenas (Tumasjan y otros, 2011; Rúas-Araujo, Puentes-Rivera y Míguez-González, 2016, Jaráiz et al., 2021).

Nuestro corpus de análisis lo constituyen 705 tuits pertenecientes a las cuentas de Pablo Iglesias (@PabloIglesias), Pedro Sánchez (@sanchezcastejon), Pablo Casado (@pablocasado_), Albert Rivera (@Albert_Rivera) y Santiago Abascal (@Santi_ABASCAL), repartidos según se muestra en la tabla 1. A fin de obtener una línea temporal para nuestro análisis, hemos agregado los tuits a nivel de día, teniendo la campaña de abril 15 días y la de noviembre $7^{5}$. Los resultados obtenidos en el análisis de sentimientos (positivos y negativos) presentes en los tuits los hemos puesto en relación con el nivel de engagement ${ }^{6}$ diario de sus cuentas de Twitter durante el mismo período.

\footnotetext{
${ }^{3}$ Se trata de los candidatos de Vox (Santiago Abascal), PP (Pablo Casado), Ciudadanos (Albert Rivera), PSOE (Pedro Sánchez) y Unidas Podemos (Pablo Iglesias), ordenados en esta nota de la posición más a la derecha a la posición más a la izquierda de la escala ideológica.

${ }^{4}$ Toda la información incluida en nuestro análisis son datos secundarios obtenidos de datos públicos de Twitter. Se ha seleccionado una muestra de tuits para cada campaña (en abril todos los tuits emitidos entre el 15 y el 27 de abril, y los emitidos entre el 4 y el 9 de noviembre). Para el análisis hemos utilizado el software Linguistic Inquiry and Word Count (LIWC) en su versión 2015 y el diccionario español del año 2007 con 7.515 palabras. Este software, desarrollado por J. Pennebaker, R. Booth y M. Francis en la Universidad de Austin (Texas), evalúa, con un enfoque semántico, cognitiva y emocionalmente textos mediante una categorización psicológica y estructural a través de 70 variables de carácter lingüístico, incluyendo procesos de carácter psicológico mediante la medición de emociones positivas y negativas.

${ }^{5}$ Se trata de todos los tuits emitidos por los candidatos durante ese período.

${ }^{6}$ Dicho indicador de engagement se obtiene calculando la media de interacciones diarias generadas por los tuits emitidos por los candidatos, partida por el número de seguidores del mismo día, durante el período de tiempo seleccionado.
} 
Tabla 1. Número de tuits analizados y media de tuits emitidos por cada uno de los líderes en las dos campañas electorales del 2019.

\begin{tabular}{|l|c|c|c|c|}
\cline { 2 - 5 } \multicolumn{1}{c|}{} & Abr-19 & $\begin{array}{c}\text { Media diaria } \\
\text { tuits emitidos }\end{array}$ & Nov-19 & $\begin{array}{c}\text { Media diaria } \\
\text { tuits emitidos }\end{array}$ \\
\hline Pablo Iglesias & 119 & 9,47 & 60 & 10,00 \\
\hline Pedro Sánchez & 107 & 8,20 & 69 & 11,75 \\
\hline Pablo Casado & 86 & 7,40 & 56 & 10,50 \\
\hline Albert Rivera & 65 & 5,80 & 37 & 6,50 \\
\hline Santiago Abascal & 74 & 6,53 & 30 & 5,25 \\
\hline Total/Media & 451 & 7,48 & 252 & 8,80 \\
\hline
\end{tabular}

Fuente: elaboración propia

La producción de tuits es mayor en la izquierda que en la derecha, y en la extrema derecha no parece especialmente relevante; pero si comparamos el volumen de tuits con los niveles de audience engagement asociado a los líderes en cada día de la campaña nos daremos cuenta de dos cosas: la primera que el engagement asociado a Abascal está muy por encima de cualquier otro, especialmente en abril; y que en ambas convocatorias, los niveles de engagement de Abascal y Casado son superiores a los otros candidatos. Dicho de otro modo, la izquierda emite más tuits, pero la derecha tiene mayores niveles de compromiso, lo cual lleva a pensar que el compromiso, al menos en su expresión primaria y cuantitativa, sigue la misma pauta que la identificación partidista, donde la derecha tiene más votantes identificados que la izquierda.

Tabla 2. Nivel de audience engagement de cada candidato durante la campaña electoral de abril de 2019

\begin{tabular}{|l|c|c|c|c|c|}
\hline & $\begin{array}{c}\text { Eng. } \\
\text { Abascal }\end{array}$ & $\begin{array}{c}\text { Eng. } \\
\text { Casado }\end{array}$ & $\begin{array}{c}\text { Eng. } \\
\text { Rivera }\end{array}$ & $\begin{array}{c}\text { Eng. } \\
\text { Sánchez }\end{array}$ & $\begin{array}{c}\text { Eng. } \\
\text { Iglesias }\end{array}$ \\
\hline Día 1 & 31,56 & 4,74 & 0,91 & 4 & 1,08 \\
\hline Día 2 & 45,89 & 6,55 & 1,86 & 1,4 & 1,85 \\
\hline Día 3 & 16,97 & 3,67 & 0,39 & 1,22 & 0,75 \\
\hline Día 4 & 15,74 & 5,86 & 1,14 & 1,89 & 1,38 \\
\hline Día 5 & 2,83 & 2,78 & 0,72 & 1,46 & 0,78 \\
\hline Día 6 & 19,79 & 3,37 & 0,48 & 2,23 & 1,08 \\
\hline Día 7 & 25,23 & 6,17 & 1,99 & 0,91 & 0,85 \\
\hline Día 8 & 28,37 & 5,82 & 2,03 & 3,98 & 1,87 \\
\hline Día 9 & 16,97 & 4,58 & 2,13 & 2,63 & 2,06 \\
\hline Día 10 & 53,83 & 5,41 & 1,72 & 2,11 & 1,75 \\
\hline Día 11 & 23,97 & 5,48 & 1,56 & 3,27 & 1,47 \\
\hline Día 12 & 32,77 & 5,6 & 1,51 & 3,19 & 4,73 \\
\hline Día 13 & 0 & 1,17 & 0,29 & 0,42 & 0 \\
\hline Media & 24,15 & 4,71 & 1,29 & 2,21 & 1,51 \\
\hline
\end{tabular}


Tabla 3. Nivel de audience engagement de cada candidato durante la campaña electoral de noviembre de 2019

\begin{tabular}{|l|c|c|c|c|c|}
\hline & $\begin{array}{c}\text { Eng } \\
\text { Abascal }\end{array}$ & $\begin{array}{c}\text { Eng } \\
\text { Casado }\end{array}$ & $\begin{array}{c}\text { Eng } \\
\text { Rivera }\end{array}$ & $\begin{array}{c}\text { Eng } \\
\text { Sánchez }\end{array}$ & $\begin{array}{c}\text { Eng } \\
\text { Iglesias }\end{array}$ \\
\hline Día 1 & 9,62 & 1,38 & 1,04 & 0,55 & 1,66 \\
\hline Día 2 & 1,1 & 4,05 & 1,23 & 2,2 & 9 \\
\hline Día 3 & 17,52 & 4,52 & 1,44 & 1,66 & 2,27 \\
\hline Día 4 & 19,73 & 2,6 & 0,86 & 1,62 & 1,74 \\
\hline Día 5 & 38,81 & 3,94 & 1,56 & 2,37 & 1,19 \\
\hline Día 6 & 8,23 & 1,64 & 0 & 0 & 1,22 \\
\hline Media & 15,84 & 3,02 & 1,02 & 1,40 & 2,85 \\
\hline
\end{tabular}

La limitada conectividad de Twitter a la que hacía referencia Conover (2011), se muestra de forma muy clara en abril de 2019, cuando se observa la correlación entre el engagement de los líderes de derecha, por un lado, y el de los líderes de izquierda, por otro, mientras el líder de Ciudadanos correlaciona con ambos grupos. Esta lectura, aparentemente nítida, no es tan clara en las elecciones de noviembre, donde la campaña es más corta y los temas más indefinidos; lo cual apunta a que el eje ideológico es solo uno de los focos que limita la conectividad, en la medida en que marca la agenda, que es la que produce el aumento o disminución del compromiso dentro de un espacio ideológico y la que genera la homogeneidad y limita la conectividad en las redes. Y por eso, mientras las elecciones de abril responden al criterio expresado por Conover, en las de noviembre, la mayor correlación se produce entre el engagement de Casado y el de Sánchez $(\mathrm{r}=0,814)$; porque mientras en abril la agenda se centraba en quien dominaba la derecha española, una competición intra-bloque, en noviembre, la agenda se centraba en quien se convertía en presidente, una competición inter-bloques. Es la competición y no la ideología la que incentiva el compromiso, de tal modo que a mayor nivel de competición mayor nivel de compromiso y viceversa. Nuestros datos refrendan la idea de una limitada conectividad, pero no la vinculan exclusivamente a la proximidad ideológica, sino al espacio de competición que se prioriza en cada momento. 
Tabla 4. Coeficiente de correlación de Pearson entre las emociones en los tuits emitidos por los políticos en la campaña de las elecciones generales de abril de 2019 y el nivel de engagement de los usuarios de Twitter.

\begin{tabular}{|c|c|c|c|c|c|c|c|c|c|c|c|c|c|c|}
\hline & $\begin{array}{l}\text { EmoPos } \\
\text { Abascal }\end{array}$ & $\begin{array}{c}\text { EmoNeg } \\
\text { Abascal }\end{array}$ & $\begin{array}{c}\text { ENG } \\
\text { Abascal }\end{array}$ & $\begin{array}{l}\text { EmoPos } \\
\text { Casado }\end{array}$ & $\begin{array}{c}\text { EmoNeg } \\
\text { Casado }\end{array}$ & $\begin{array}{l}\text { ENG } \\
\text { Casado }\end{array}$ & $\begin{array}{c}\text { EmoPos } \\
\text { Rivera }\end{array}$ & $\begin{array}{c}\text { EmoNeg } \\
\text { Rivera }\end{array}$ & $\begin{array}{c}\text { ENG } \\
\text { Rivera }\end{array}$ & $\begin{array}{l}\text { EmoPos } \\
\text { Sánchez }\end{array}$ & $\begin{array}{l}\text { EmoNeg } \\
\text { Sánchez }\end{array}$ & $\begin{array}{c}\text { ENG } \\
\text { Sánchez }\end{array}$ & $\begin{array}{l}\text { EmoPos } \\
\text { Iglesias }\end{array}$ & $\begin{array}{c}\text { EmoNeg } \\
\text { Iglesias }\end{array}$ \\
\hline EmoPosAbascal & 1 & & & & & & & & & & & & & \\
\hline ENG Abascal & $-0,515$ & $-0,132$ & 1 & & & & & & & & & & & \\
\hline EmoPos Casado & 0,143 & 0,038 & $-0,266$ & 1 & & & & & & & & & & \\
\hline EmoNeg Casado & $-0,329$ & 0,118 & 0,414 & $-0,248$ & 1 & & & & & & & & & \\
\hline EmoPos Rivera & $-0,177$ & $-0,392$ & 0,204 & 0,279 & 0,251 & 0,195 & 1 & & & & & & & \\
\hline EmoNeg Rivera & 0,459 & 0,012 & $-0,443$ & $-0,032$ & $-0,338$ & $-0,467$ & $-0,544$ & 1 & & & & & & \\
\hline ENG Rivera & $-0,432$ & 0,227 & 0,343 & $-0,046$ & 0,189 &, $754^{* *}$ & 0,176 & $-0,450$ & 1 & & & & & \\
\hline EmoPos Sánchez & 0,456 & 0,126 & $-0,504$ & 0,408 & $-0,537$ &,$- 663^{*}$ & $-0,133$ &, $574^{*}$ & $-0,275$ & 1 & & & & \\
\hline EmoNeg Sánchez & $-0,424$ & 0,007 & 0,187 &,$- 639^{*}$ & 0,011 & 0,174 & $-0,037$ & $-0,314$ & 0,161 & $-0,376$ & 1 & & & \\
\hline ENG Iglesias & $-0,137$ & $-0,264$ & 0,420 & 0,482 & 0,192 &, $556^{*}$ & 0,533 & $-0,526$ & 0,529 & $-0,288$ & $-0,187$ &, $553^{*}$ & 0,111 & $-0,482$ \\
\hline
\end{tabular}

Fuente: elaboración propia a partir de los datos de Twitter. **. La correlación es significativa en el nivel 0,01 (bilateral). *. La correlación es significativa en el nivel 0,05 (bilateral).

Tabla 5. Coeficiente de correlación de Pearson entre las emociones en los tuits emitidos por los políticos en la campaña de las elecciones generales de noviembre de 2019 y el nivel de engagement de los usuarios de Twitter.

\begin{tabular}{|c|c|c|c|c|c|c|c|c|c|c|c|c|c|c|}
\hline & $\begin{array}{l}\text { EmoPos } \\
\text { Abascal }\end{array}$ & $\begin{array}{c}\text { EmoNeg } \\
\text { Abascal }\end{array}$ & $\begin{array}{c}\text { ENG } \\
\text { Abascal }\end{array}$ & $\begin{array}{c}\text { EmoPos } \\
\text { Casado }\end{array}$ & $\begin{array}{c}\text { EmoNeg } \\
\text { Casado }\end{array}$ & $\begin{array}{c}\text { ENG } \\
\text { Casado }\end{array}$ & $\begin{array}{c}\text { EmoPos } \\
\text { Rivera }\end{array}$ & $\begin{array}{c}\text { EmoNeg } \\
\text { Rivera }\end{array}$ & $\begin{array}{c}\text { ENG } \\
\text { Rivera }\end{array}$ & $\begin{array}{l}\text { EmoPos } \\
\text { Sánchez }\end{array}$ & $\begin{array}{l}\text { EmoNeg } \\
\text { Sánchez }\end{array}$ & $\begin{array}{c}\text { ENG } \\
\text { Sánchez }\end{array}$ & $\begin{array}{l}\text { EmoPos } \\
\text { Iglesias }\end{array}$ & $\begin{array}{l}\text { EmoNeg } \\
\text { Iglesias }\end{array}$ \\
\hline EmoPosAbascal & 1 & & & & & & & & & & & & & \\
\hline EmoNeg Abascal & $-0,472$ & 1 & & & & & & & & & & & & \\
\hline EmoPos Casado &, $964^{* *}$ & $-0,439$ & $-0,439$ & 1 & & & & & & & & & & \\
\hline EmoNeg Casado & $-0,020$ & $-0,162$ &,$- 957^{* *}$ & 0,219 & 1 & & & & & & & & & \\
\hline EmoNeg Rivera & $-0,227$ & $935^{*}$ & $-0,097$ & 0,331 & 0,150 & $-0,839$ &,$- 881^{*}$ & 1 & & & & & & \\
\hline ENG Rivera &,$- 916^{*}$ & 0,387 & 0,118 &,$- 916^{*}$ & $-0,212$ & 0,649 & 0,656 & $-0,314$ & 1 & & & & & \\
\hline EmoPos Sánchez & $-0,259$ & 0,824 & 0,371 & $-0,128$ & $-0,243$ & $-0,309$ & $-0,365$ & 0,698 & 0,220 & 1 & & & & \\
\hline EmoNeg Sánchez & $-0,787$ & 0,353 & $-0,747$ & 0,850 & 0,827 & $-0,319$ & $-0,339$ & 0,547 & $-0,101$ & 0,190 & 1 & & & \\
\hline ENG Sánchez & $-0,713$ & $-0,097$ & 0,462 & $-0,790$ & $-0,318$ &, $814^{*}$ & 0,809 &,$- 883^{*}$ & 0,509 & $-0,589$ & $-0,585$ & 1 & & \\
\hline
\end{tabular}

Fuente: elaboración propia a partir de los datos de Twitter. **. La correlación es significativa en el nivel 0,01 (bilateral). *. La correlación es significativa en el nivel 0,05 (bilateral).

La emisión de tuits permite controlar la estrategia, utilizar el lenguaje emocional para producir efectos sobre los seguidores, y es el modo más nítido de conocer el tipo de vínculos que se establecen entre un líder y sus seguidores a través del lenguaje emocional que construye las comunidades. En las elecciones de abril de 2019, el caso más claro es sin duda el de Casado, que refuerza el engagement a través de la producción de tuits con 
una semántica emocional negativa, de tal modo que cuanto más negativas sean las emociones que se expresan en sus tuits, mayor engagement generan, como muestra la correlación. Es cierto que en estas elecciones, Casado se sostenía por la crítica a Sánchez, y por lo tanto, las emociones negativas eran la base de un discurso que planteaba la alternativa desde la negatividad hacia el otro.

Esta forma directa de correspondencia entre la producción emocional de discurso a través de las redes y la generación de engagement hacia el propio sujeto que produce el discurso ha sido ampliamente estudiada. En este caso, son las emociones negativas las que favorecen el engagement, coincidiendo con la mayoría de investigadores, pero lo cierto es que eso depende mucho de la estrategia, y del framing emocional en el que se enmarca la estrategia. Nuestro trabajo quiere, además, avanzar en otra dirección exploratoria, a saber, al mismo tiempo que la producción semántica de emociones negativas por parte de Casado favorece su engagement, la producción de discurso emocionalmente positivo por parte de Abascal correlaciona con la disminución del engagement de Casado. Y es aquí donde está la verdadera novedad de este trabajo, al intuir que la relación entre discurso emocional y engagement hacia un líder no es exclusivamente endógena, sino que puede obedecer también a elementos exógenos debido a la presencia de comunidades compartidas; es decir, miembros que forman parte de varias comunidades o están en proceso de tránsito entre comunidades. No olvidemos que, como señalamos antes, en las elecciones de abril de 2019 la competición se planteaba en términos de quien lideraba la derecha, y los realineamientos de los electores de derechas propiciaban el crecimiento de VOX y Ciudadanos. Lo mismo ocurre con las emociones positivas contenidas en los mensajes de Sánchez y el engagement de Casado, a medida que disminuyen las primeras crece el segundo, porque éste es otro eje de la competición.

La debacle de Rivera en las elecciones de noviembre también aparece marcada en este trabajo a través de una intensa correlación inversa entre la producción de emociones positivas en las cuentas de Casado y de Abascal y el engagement al propio Rivera. Son las relaciones más intensas de este estudio y muestran, con dicha intensidad, hasta qué punto las comunidades en tránsito permiten la existencia de relaciones exógenas entre dos comunidades digitales ${ }^{7}$.

\section{CONCLUSIONES}

El presente artículo contribuye a apuntalar la evidencia sobre la relación entre el nivel de compromiso alcanzado por los mensajes de los políticos en las redes sociales y la carga emocional presente en los mismos. Nuestro análisis de los tuits emitidos por los principales candidatos en las elecciones generales de abril y noviembre de 2019 en España, nos ha permitido comprobar algunas de las hipótesis planteadas. En primer lugar, parece evidente que la extrema derecha optimiza el uso de las redes sociales para generar engagement, siendo su líder el que alcanza un mayor nivel de compromiso en ambas campañas electorales, con gran diferencia sobre el resto de líderes. Esto es especialmente interesante en un momento de elevada polarización política, y en el que VOX es el partido que más crece, lo que nos sugiere la importancia del estudio del compromiso político y

\footnotetext{
${ }^{7}$ Algunas de las relaciones que se muestran en estas tablas son expresión del crecimiento común de los líderes o de estrategias semánticas compartidas, por ejemplo, frente a las utilización de una semántica emocional negativa en las elecciones de abril, por parte de Casado, en noviembre, la producción emocional es netamente positiva en semejanza a la de Abascal; pero eso también tiene que ver con que mientras en abril Casado trataba de frenar su caída con una estrategia defensiva, en noviembre su estrategia es de ataque y su framing emocional positivo.
} 
su posible efecto en el comportamiento electoral, aunque esto queda totalmente fuera del alcance de esta investigación.

Además, la limitada conectividad de los usuarios de Twitter y su agrupación en redes de corte ideológico, parece clara en las elecciones de abril de 2019, corroborando estudios previos, pero no así en la campaña de noviembre, en la que la agenda podría tener un papel especialmente relevante, trasladando el diálogo en las redes de la competición intra-bloque a la disputa de la Presidencia del Gobierno. Los datos nos indican que cuanto mayor es la competitividad entre líderes, mayor es el nivel de compromiso que se genera, y que la limitada conectividad no se vincula únicamente a la proximidad ideológica, sino al espacio de la competición, aportando un carácter dinámico a la producción del compromiso político.

Finalmente, ante la disyuntiva sobre si son las emociones positivas o negativas las que generan más engagement, aunque las negativas parecen tener más impacto cuando la relación es endógena, nuestro trabajo apunta a que la relación entre discurso emocional y engagement hacia un líder puede obedecer también a elementos exógenos, al menos cuando hay comunidades compartidas, y en este caso, las emociones positivas cobran mayor fuerza.

\section{REFERENCIAS BIBLIOGRÁFICAS}

Aldrich, John. H., Rachel K. Gibson, Marta Cantijoch y Tobías Konitzer. 2016. «Getting out the vote in the social media era: Are digital tools changing the extent, nature and impact of party contacting in elections?». Party Politics, 222: 165-178. https://doi.org/10.1177/1354068815605304

Anduiza, Eva, Michael J. Jensen y Laia Jorba. 2012. Digital Media and Political Engagement Worldwide. A Comparative Study. Cambridge: Cambridge University Press.

Barrett, Marthyn y Bruna Zani. 2014. Political and Civic Engagement: Multidisciplinary Perspectives. Londres: Routledge.

Baumgartner, Jody C. y Jonathan S. Morris. 2010. «MyFaceTube politics: social networking web sites and political engagement of young adults». Social Science Computer Review, 281: 24-44. https://doi.org/10.1177/0894439309334325

Bazarova, Natalya. N., Yoon H. Choi, Victoria Schwanda Sosik, Dan Cosley y Janis Whitlock. 2015. «Social sharing of emotions on Facebook: Channel differences, satisfaction, and replies». Proceedings of the 18th ACM conference on computer supported cooperative work \& social computing, 154-164.

Berger, Jonah y Katherine L. Milkman. 2012. «What makes online content viral?» Journal of Marketing Research, 49: 192-205. https://doi.org/10.1509/jmr.10.0353

Bhattacharya, Sanmitra, Chao Yang, Padmini Srinivasan y Bob Boynton. 2015. «Perceptions of presidential candidates' personalities in Twitter». J. Assoc. Inform. Technol, 67: 249-267. https://doi.org/10.1002/asi.23377

Bimber, Bruce. 1999. «The Internet and Citizen Communication with Government: Does the Medium Matter». Political Communication, 16: 409-428. https://doi.org/10.1080/105846099198569

Bimber, Bruce. 2001. «Information and political engagement in America: The search for effects of information technology at the individual level». Political Research Quarterly, 54: 53-67. https://doi.org/10.1177/106591290105400103

Bond, Robert. M., Christopher J. Fariss, Jason J. Jones, Adam D. Kramer, Cameron Marlow, Jaime E. Settle y James H. Fowler. 2012. «A 61-million-person 
experiment in social influence and political mobilization». Nature, 4897415: 295298.

Brady, William J., Julian A. Wills, John T. Jost, Joshua A. Tucker, y Jay J. Van Bavel. 2017. «Emotion shapes the diffusion of moralized content in social networks». Proceedings of the National Academy of Sciences, 11428: 7313-7318.

Cantijoch, Marta, David Cutts y Rachel Gibson. 2015. «Moving slowly up the ladder of political engagement: A "spill-over" model of Internet participation». The British Journal of Politics and International Relations, 181: 26-48. https://doi.org/10.1111/1467-856X.12067

Ceron, Andrea y Giovanna d'Adda. 2016. «E-campaigning on Twitter: The effectiveness of distributive promises and negative campaign in the 2013 Italian election». New Media \& Society, 18: 1935-1955. https://doi.org/10.1177/1461444815571915

Conover, Michael D., Jacob Ratkiewicz, Matthew Francisco, Bruno Goncalves, Filippo Menczer y Alessandro Flammini. 2011. «Political polarization on Twitter». Proceedings of the ICWSM: Fifth International AAAI Conference on Weblogs and Social Media. Barcelona, Spain.

Cvijikj, Irena P. y Florian Michahelles. 2013. «Online engagement factors on Facebook brand pages». Social Network Analysis and Mining, 3: 843-861.

Dahlgren, Peter. 2009. Media and Political Engagement: Citizens, Communication, and Democracy. Cambride: Cambridge University Press.

D’Andrea, Alessia, Fernando Ferri, Patrizia Grifoni y Tiziana Guzzo, 2015. «Approaches, tools and applications for sentiment analysis implementation». Int. J. Comput. Appl, 125: 26-33. https://doi.org/10.5120/ijca2015905866

Eckstein, Katharina, Peter Noacka y Burkhard Gniewosz. 2012. «Attitudes toward political engagement and willingness to participate in politics: Trajectories throughout adolescence». Adolesc, 35: 485-495. https://doi.org/10.1016/j.adolescence.2011.07.002

Ekman, Joakim y Erik Amnå. 2012. «Political participation and civic engagement: Towards a new typology». Human Affairs, 223: 283-300. https://doi.org/10.2478/s13374- 012-0024-1

Ellison Nicole B., Charles Steinfield y Cliff Lampe. 2007. «The benefits of Facebook "friends": social capital and college students'use of online social network sites». Journal of Computer-Mediated Communication, 124: 1143-1168. https://doi.org/10.1111/j.1083-6101.2007.00367.x

Enli, Gunn. 2017. «Twitter as Arena for the Authentic Outsider: Exploring the Social Media Campaigns of Trump and Clinton in the 2016 US Presidential Election». European Journal of Communication, 32: 50-61. https://doi.org/10.1177/0267323116682802

Entman, Robert M. 1993. «Framing: Toward clarification of a fractured paradigm». Journal of Communication, 43: 51-58. https://doi.org/10.1111/j.14602466.1993.tb01304.x

Feller, Albert, Matthias Kuhnert, Timm O. Sprenger, Isabell M. Welpe, Isabell. 2011. «Divided they tweet: The network structure of political microbloggers and discussion topics». In Proceedings of the Fifth Intl AAAI Conf on weblogs and social media. (pp. 474-477). Recuperado de https://ojs.aaai.org/index.php/ICWSM/article/view/14150

Fishbein, Martin y Icek Ajzen. 1975. Belief, Attitude, Intention, and Behavior: An Introduction to Theory and Research. Reading: Addison-Wesley.

Gerbaudo, Paolo, Federico Marogna y Chiara Alzetta. 2019. "When "Positive Posting" Attracts Voters: User Engagement and Emotions in the 2017 UK Election 
Campaign on Facebook». Social media + Society, 1-11. https://doi.org/10.1177/2056305119881695

Gibson, Rachel y Marta Cantijoch. 2013. «Conceptualizing and measuring participation in the age of the Internet: Is online political engagement really different to offline?». The Journal of Politics, 753: 701-716. https://doi.org/doi:10.1017/S0022381613000431

Gil de Zúñiga, Homero, Nakwon Jung y Sebastián Valenzuela. 2012. «Social media use for news and individuals' social capital, civic engagement and political participation». Journal of Computer-Mediated Communication, 17: 319-336. https://doi.org/doi:10.1111/j.1083-6101.2012.01574.x

Gil de Zúñiga, Homero y Sebastián Valenzuela. 2011. «The mediating path to a stronger citizenship: Online and offline networks, weak ties and civic engagement». Communication Research, 383:

$397-421$. https://doi.org/10.1177/0093650210384984

Gordon, Steven L. 1981. «The Sociology of Sentiments and Emotions». En Social Psychology: Sociological Perspectives, eds. Morris Rosenberg y Ralph H. Turner (pp. 562-569). New York: Routledge.

Hall, Cheryl. 2005. The Trouble with Passion: Political Theory Beyond the Reign of Reason. New York: Routledge.

Jaráiz-Gulías, Erika, María Pereira-López y José Manuel Rivera-Otero. 2020. «Análisis de emociones originadas por las publicaciones de Twitter de los candidatos en las elecciones generales de Bolivia y España en 2019». Journal of Iberian and Latin American Research, 26(3): 371-388.

Jung, Nakwon, Yonghwam Kim, Homero Gil de Zúñiga. 2011. «The mediating role of knowledge and efficacy in the effects of communication on political participation». Mass Communication and Society, 14(4): 407-430. https://doi.org/10.1080/15205436.2010.496135

Kenski, Kate, Bruce W. Hardy y Kathleen H. Jamieson. 2010. The Obama Victory: How Media, Money, and Messages Shaped the 2008 Election. Oxford: Oxford University Press.

Kreis, Ramona. 2017. «The tweet politics of president Trump». Journal of Language and Politics, 4: 607-618. https://doi.org/10.1075/jlp.17032.kre

Krueger, Brian. 2002. «Assessing the potential of Internet political participation in the United States: A resource approach». American Politics Research, 305: 476-498. https://doi.org/10.1177/1532673x02030005002

Krueger, Brian. 2010. «Opt in or tune out: Email mobilization and political participation». International Journal of E-Politics IJEP, 14: 55-76. https://doi.org/10.4018/jep.2010100104

Lee, Dokyum, Kartik Hosanagar y Harikesh Nair. 2014. The effect of social media marketing content on consumer engagement: Evidence from Facebook. Stanford: Stanford Graduate School of Business.

Lee, Jayeon y Weiai Xu. 2018. «The more attacks, the more retweets: Trump's and Clinton's agenda setting on Twitter». Public Relations Review, 44: 201-213. https://doi.org/10.1016/j.pubrev.2017.10.002

Lilleker, Darr. 2013. «Empowering the citizens? Political communication, co-production and the harnessed crowd». En The media, political participation and empowerment, eds. Scullion, Richard, Roman Gerodimos, Daniel Jackson y Darren Lilleker (pp. 24-38). Londres: Routledge.

Liu, Dilin y Lei Lei. 2018. «The appeal to political sentiment: An analysis of Donald Trump's and Hillary Clinton's speech themes and discourse strategies in the 2016 
US presidential election». Discourse, Context \& Media, 25: 143-152. https://doi.org/10.1016/j.dcm.2018.05.001

Macková, Alena. 2015. «Czech politicians go online: Is this e-democracy or just a campaign move? ». En Living in the digital age, eds. Lorentz, Pascaline, David Smahel, Monika Metykova y Michelle F. Wright (pp. 164-180). Brno: Masarykova univerzita.

Malhotra, Neil, Melissa R. Michelson, Todd Rogers y Ali Adam Valenzuela. 2011. «Text messages as mobilization tools: The conditional effect of habitual voting and election salience». American Politics Research, 39: 664-681. http:dx.doi.org/10.1177/1532673X11398438

Magalhães, Pedro C. 2005. «Disaffected Democrats: Political Attitudes and Political Action in Portugal». West European Politics, 285: 973-991. https://doi.org/10.1080/01402380500310626

Murthy, Dhiraj. 2015. «Twitter and elections: are tweets, predictive, reactive, or a form of buzz?». Inform. Commun. Soc. 18: 816-831. https://doi.org/10.1080/1369118X.2015.1006659

McCartney, Allison, Elizabeth Bennion y Dick Simpson. 2013. Teaching Civic Engagement: From Student to Active Citizen, Washington: American Political Science Association.

Morozov, Evgeny. 2011. The Net Delusion: How Not to Liberate the World. Londres: Penguin.

Nabi, Robin. 2003. «Exploring the Framing Effects of Emotion: Do Discrete Emotions Differentially Influence Information Accessibility, Information Seeking, and Policy Preference?». Communication Research, 30: 224-247 https://doi.org/10.1177/0093650202250881

Nave, Nir Noon, Limor Shifman y Keren Tenenboim-Weinblatt. 2018. «Talking it personally: Features of successful political posts on Facebook». Social Media+ Society, 43: 1-12. https://doi.org/10.1177/2056305118784771

Neuman, W. Russel, George E. Marcus y Michael B. Mackuen. 2018. «Hardwired for News: Affective Intelligence and Political Attention». Journal of Broadcasting \& $\begin{array}{lllll}\text { Electronic } & \text { Media, } & 62 & \text { (4): }\end{array}$ https://doi.org/10.1080/08838151.2018.1523169

Norris, Pippa. 2001. «A Virtuous Circle? The Impact of Political Communications in Post-Industrial Democracies». En Challenges to Democracy, eds. Dowding, Keith, Jim Hughes y Helen Margetts (pp. 100-117). Londres: Palgrave MacMillan.

Norris, Pippa. 2003. «Preaching to the converted? Pluralism, participation and party websites». Party Politics, 91: 21-45. https://doi.org/10.1177/135406880391003

Norris, Pippa y John Curtice. 2008. «Getting the Message Out: A Two-Step Model of the Role of the Internet in Campaign Communication Flows During the 2005 British General Election». Journal of Information Technology \& Politics, 4: 3-13. https://doi.org/10.1080/19331680801975359

Redlawsk, David P. 2006. Feeling Politics. Emotion in Political Information Processing. New York: Palgrave Macmillan.

Rosenstone, Steven y John Hansen. 1993. Mobilization, participation and democracy in America. New York: Longman.

Rúas-Araujo, José, Puentes-Rivera, Iván y María Isabel Míguez-González. 2016. «Capacidad predictiva de Twitter, impacto electoral y actividad en las elecciones al Parlamento de Galicia: un análisis con la herramienta LIWC». Observatorio, 10(2): 55-87. https://doi.org/10.15847/obsOBS1022016893 
Sahly, Abdulsamad, Chun Shao y Kyounghee Hazel Kwon. 2019. «Social Media for Political Campaigns: An examination of Trump's and Clinton's Frame Building and Its Effect on Audience Engagement». Social Media \& Society, 1-13.

Savoy, Jacques. 2017. «Trump's and Clinton's style and rhetoric during the 2016 presidential election». J. Quant. Linguist, 1-22.

Skoric, Marko M., Qinfeng Zhu, Debbie Goh y Natalie Pang. 2016. «Social Media and Citizen Engagement: A Meta-Analytic Review». New Media \& Society, 18: $1817-1839$.

Stanley, Wood y Christopher Weare. 2004. «The Effects of Internet Use on Political Participation: Evidence From an Agency Online Discussion Forum». Administration $\quad \& \quad$ Society, 36 : 503-527. https://doi.org/10.1177/0095399704268503

Tang, Gary y Francis Lee. 2013. «Facebook use and political participation: The impact of exposure to shared political information, connections with public political actors, and network structural heterogeneity». Social Science Computer Review, 316: 763-773. https://doi.org/10.1177/0894439313490625

Tumasjan, Adrianik, Timm O. Sprenger, Philipp G. Sandner y Isabell M. Welpe. 2011. «Election forecasts With Twitter:How 140 characters reflect the political landscape». Social Science Computer Review, 294: 402-418. https://doi.org/10.1177/0894439310386557

Valenzuela, Sebastián, Yonghwan Kim y Homero Gil de Zúñiga. 2012. «Social networks that matter: Exploring the role of political discussion for online political participation». International Journal of Public Opinion Research, 242: 163184. https://doi.org/10.1093/ijpor/edr037

Valenzuela, Sebastián, Daniel Halpern, James E. Katz y Juan Pablo Miranda. 2019. «The Paradox of Participation Versus Misinformation: Social Media, Political Engagement, and the Spread of Misinformation». Digital Journalism, 7: 802-823. https://doi.org/10.1080/21670811.2019.1623701

Vaccari, Cristian. 2016. «Online Mobilization in Comparative Perspective: Digital Appeals and Political Engagement in Germany, Italy, and the United Kingdom». Political Communication, 341: 6988. https://doi.org/10.1080/10584609.2016.1201558

Valeriani, Augusto y Cristian Vaccari. 2017. «Political Talk on Mobile Instant Messaging Services: A Comparative Analysis of Germany, Italy, and the UK». Information Communication and Society, 21: 1715-31. https://doi.org/10.1080/1369118X.2017.1350730

Verba, Sidney, Kay Lehman Schlozman y Henry E. Brady. 1995. Voice and equality: Civic voluntarism in American politics. Cambridge: Harvard University Press.

Wise, Kevin, Paul D. Bolls y Samantha R. Schaefer. 2008. «Choosing and reading online news: How available choice affects cognitive processing». Journal of Broadcasting \& Electronic Media, 523: 69-85. https://doi.org/10.1080/08838150701820858

Yarchi, Moran, Christian Baden y Neta Kligler-Vilenchik. 2020. «Political polarization on the digital sphere: A cross-platform, over-time analysis of interactional, positional, and affective polarization on social media». Political Communication, 1-42. https://doi.org/10.1080/10584609.2020.1785067

Zillmann, Dolf. 1971. «Excitation transfer in communication-mediated aggressive behavior». Journal of Experimental Social Psychology, 7: 419-434 https://doi.org/10.1016/0022-1031(71)90075-8 